\title{
V014 AVO WALKAWAY PROCESSING - A CASE STUDY FROM THE NORTH SEA
}

KAPILA EDIRIWEERA', PHILIPPE GUERENDEL' and MARTIN GUNDEM ${ }^{2}$

' Schlumberger/GeoQuest, PO Box 330, 4001 Stavanger, Norway

${ }^{2}$ Norsk Hydro

\begin{abstract}
AVO analysis of surface seismic data has become a widely used technique in recent years. Its success is dependent on the quality of log data available for modelling the AVO at the well positions and the availability of AVO Walkaway (VSP) data surrounding the well. The AVO response observed on the CMP gathers often does not match the well response, and this mismatch may have several reasons (Amstrong, et. al., 1995). The most documented are processing-related issues, anisotropy and some geometric effects. Effective forward modelling of the AVO response from logs, and the flexibility to introduce anisotropy, make it easier to obtain reasonable surface seismic AVO responses of walkaway borehole seismic profiles around well locations. This provides a potential improvement in AVO calibration of surface seismic data (Landrø, et. al., 1995, Schultz, et. al., 1994).
\end{abstract}

This paper presents a case study of a recent AVO walkaway dataset processed for a North Sea client (Fig. 1). A total of five walkaway lines were run with Schlumberger's Combinable Seismic Imager (CSI) tool with a total survey acquisition interval of $228 \mathrm{~m}$. The horizontal coverage of 20 geophone lines (4xCSI configuration) in the well was about $100 \mathrm{~m}$. The source offset of each walkaway line was about $2.2 \mathrm{~km}$ (half the distance of an equivalent surface seismic profile) and contained about 120 shots. The walkaway lines were run parallel to each other but oblique to the well path, which was oriented approximately 61 degrees in a NE-E direction.

The study had several objectives. Firstly, to obtain a reasonable AVO response of the shale/topsand interface - which has a highly irregular surface character on the surface seismic. Secondly, to obtain a similar response from the sand/shale interface at the bottom of the reservoir sand (Heimdal sand). Beneath this interface is a thin shale layer which is underlain by high impedance Cretaceous carbonates. Thirdly, to use the information for upcoming AVO studies in the field.

The data was initially rotated based on the geometry and the incident P-wave energy to give true vertical and in-line horizontal components for each receiver. The first break times of each receiver lines were picked and the travel time curves were used to calculate the Transverse Isotropy in the medium (Miller \& Spencer, 1994). The Parametric Wavefield Decomposition technique (Esmersoy, 1990; Leaney, 1990) has been employed for wavefield separation of common geophone gathers. In this approach, two input wavefield components (vertical and in-line horizontal) are used to decompose the signal into four wavemodes - upgoing and downgoing, compressional $(\mathrm{P})$ and shear $(\mathrm{S})$. The wavemodes are modeled with respect to their polarisation and slowness, and the best fit to the real data being obtained using a non-linear least squares minimisation approach. In this technique, true amplitudes have been retained for each wavefield and each common source gather is treated independently.

The next step in the processing was a deterministic deconvolution, using the downgoing Pwavefield to deconvolve the upgoing P-wavefield. Considering the fact that the receiver array is located very close to the target zone, it is therefore valid to assume that the deconvolution will remove all of the propagation effects present in the downwaves - including multiples and attenuation. An 8-60 Hz zero-phase wavelet was chosen as the desired output of the deconvolved upgoing wavefield. The deconvolved upgoing wavefield was then used for AVO analysis.

EAGE Winter Symposium, Reservoir Geophysics: The Road Ahead - Venice Lido, Italy, 27 - 30 October 1996 
We have presented normalized AVOAVA response curves for top and bottom sand interfaces from 12 receiver lines. We compared the measured AVO/AVA responses with those of the modelbased data and the agreement was very good. We also produced a phase slowness curve using TIV parameter inversion (Fig.2) for the shale layer above the reservoir sand - and it was found to have an insignificant amount of anisotropy. Our model-based data versus measured-data comparison revealed that the flat layer velocity model used for the study is valid for the AVO responses measured at the well, and could be used for surface seismic AVO studies greater than $2 \mathrm{~km}$ across this well (Fig. $3 \& 4$ ).

\section{Conclusions}

The log derived lithological boundaries and their petrophysical characteristics often contain very high quality information which can be used very effectively to model the subsurface. The Walkaway and Walkabove VSP (vertical incidence) datasets bridge the gap between the surface seismic and log data, providing downhole seismic measurements from which most propagation effects can successfully be removed. Since the VSP Fresnel zones are far smaller than those on the surface seismic data, the VSP is more likely to match the logs.

The anisotropy information available from the walkaway dataset is of high quality, and this verifies the mismatch observed between surface seismic AVO responses and those modeled from logs. It is clear from this study that the combination of AVO/TI measurement from VSP and logbased anisotropic modelling will provide a good methodology for calibrating AVO responses observed on surface seismic data near to wells.

\section{References}

Amstrong, P. N., Chmela, W. and Leaney, W. S. 1995. Avo calibration using borehole data, 56th International EAEG meeting (Vienna) extended abstracts, G028.

Esmersoy, C., 1990, Inversion of P and SV waves from multicomponent offset vertical seismic profiles, Geophysics 57, 1194-1198.

Landrø, M., Buland, A. and D'Angelo, R. 1995. Target-oriented AVO inversion of data from Valhall and Hod fields, The Leading Edge, Vol 14, 8, 855-861.

Leaney, W. S., 1990, Parametric Wavefield Decomposition and applications, 60th Intern. SEG meeting (San Fransisco) expanded abstracts, 105-109.

Miller, D. E. and Spencer, C., 1994, An exact inversion for anisotropic moduli from phase slowness data, Jour. Geophys. Res., 99, 21651-21657. 


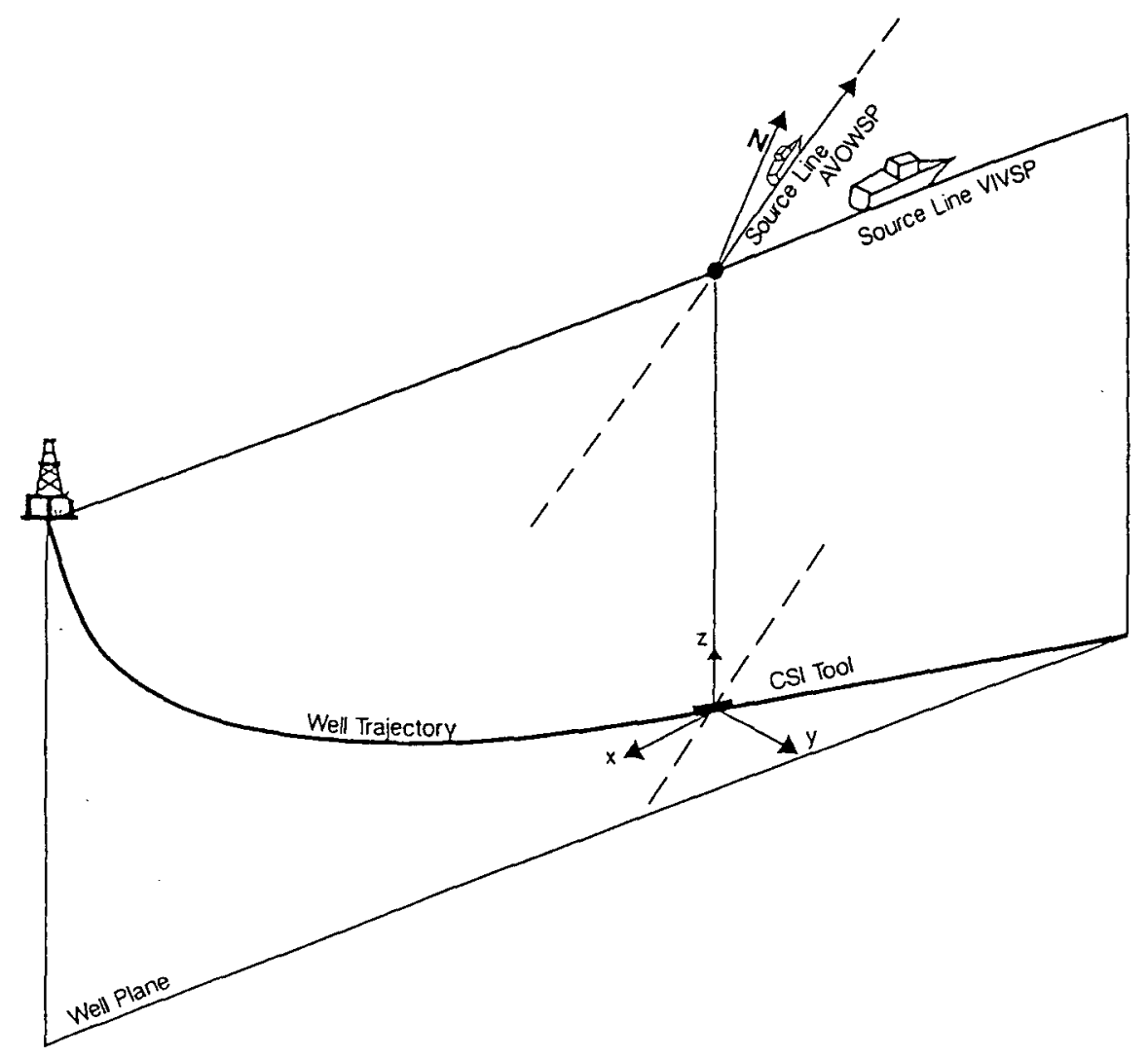

Figure 1 Schematic showing VIVSP and AVOWSP survey geometry

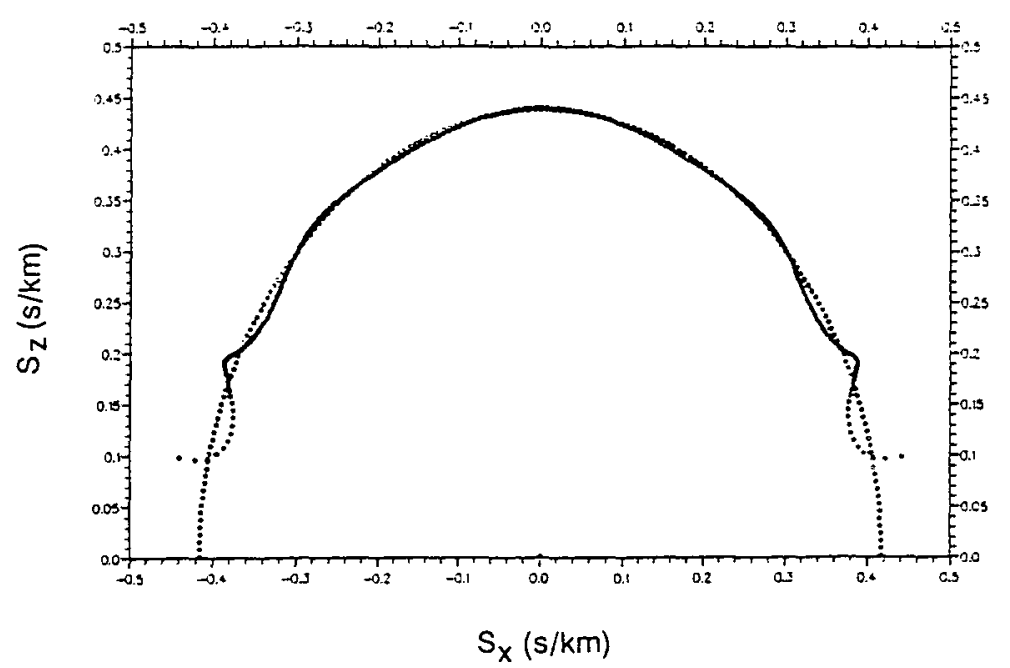

Figure 2 TIV modelled and walkaway-derived slowness curves (Receiver 7) 

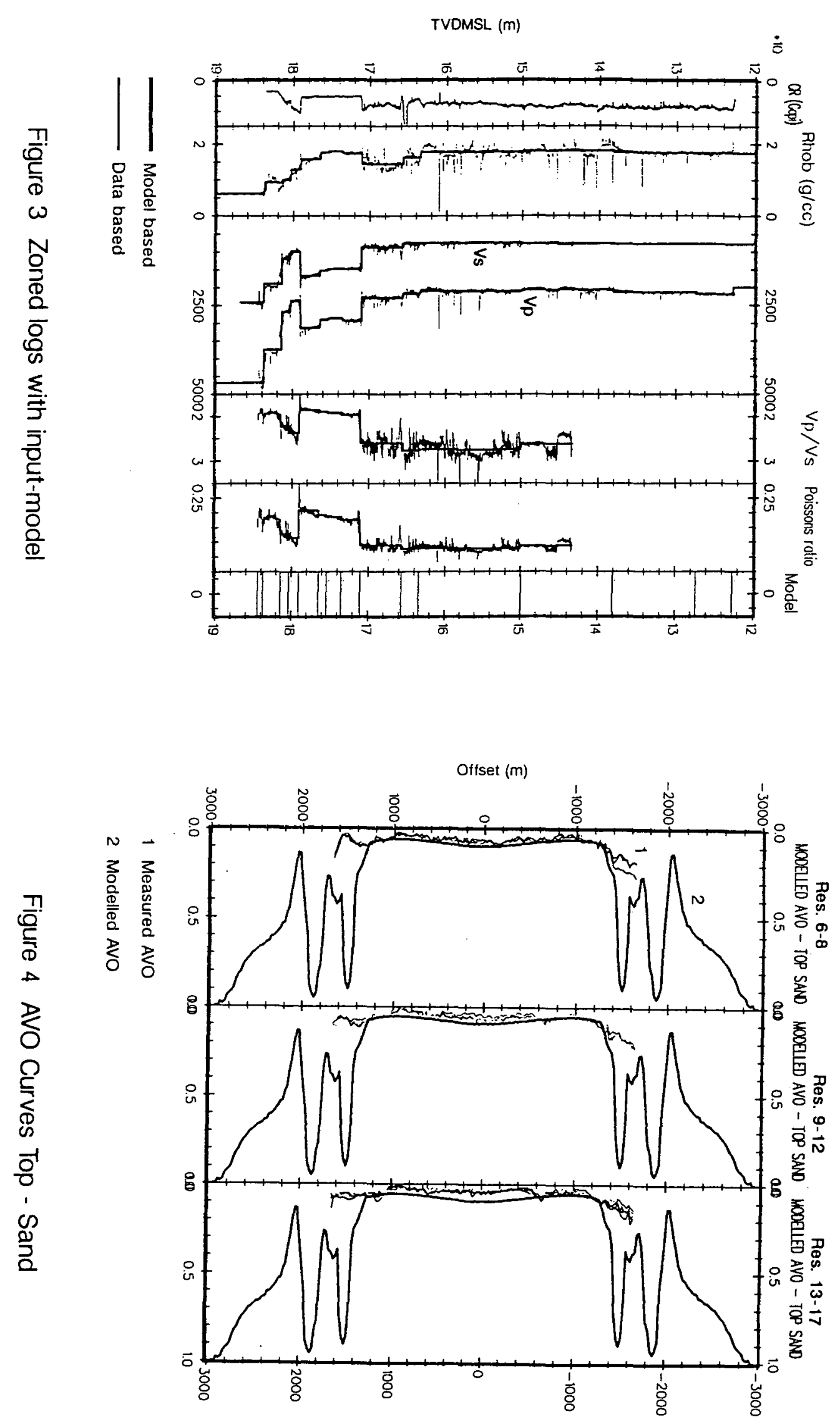